Version 20 August 2014

This is a pre-edited version of an article published in Science as Culture:

Grommé, Francisca. 2015. "Turning Aggression into an Object of Intervention: Tinkering in a Crime Control Pilot Study." Science as Culture 24 (2): 227-47.

doi:10.1080/09505431.2014.992331.

The published article can be found on:

http://www.tandfonline.com/doi/abs/10.1080/09505431.2014.992331

\title{
Turning Aggression into an Object of Intervention: Tinkering in a Crime Control Pilot Study
}

\author{
FRANCISCA GROMMÉ
}

Department of Anthropology, University of Amsterdam, The Netherlands

\begin{abstract}
Real-world experiments that test new technologies can affect policy and practice by introducing new objects of intervention through tinkering; the ad hoc work of realigning relations in the face of frictions, surprises, and disturbances that occur when introducing a technology. In a pilot study on aggression detection, tinkering moved aggression in and out of the human body. In the end, the pilot study constituted aggression as a set of acousticphysical variables representing the aroused human body, alongside other signals of aggression. How aggression as an object intervention was established by tinkering is relevant because it involved inclusions and exclusions by the authorities who identified aggression, the methods they applied, and mandate for intervention. A focus on relations that are tinkered with in a real-world experiment permits critical engagement with this format. Although the format of experimenting outside of the laboratory is credited with producing knowledge about a technology's 'actual' performance, actors and events at the pilot study location were made only selectively relevant. Analyses of real-world experiments should therefore explain how experiments selectively make the world relevant, giving only particular objects of intervention a truth status.
\end{abstract}

KEY WORDS: Aggression; Real-world experiment; Sound; Pilot study; Crime control; Tinkering

\section{Introduction}

In 2010 and 2011, Bus Station S in a large Dutch city was the site of a pilot study on the acoustic detection of aggression. ${ }^{1}$ The technology used alerted a nearby police station if a person sounded aggressive. This experiment appears to elucidate some key issues surrounding experiments with technology in operational settings. In the 
academic literature, such efforts are referred to as real-world experiments, policy experiments, or regulatory experiments.

As noted in the Netherlands and other European and Anglo-American countries, an experimental attitude has become an integrated part of crime control. ${ }^{2}$ Science and technology thus play important roles in this changing field. Not only are knowledge and technologies produced and tested, scientific activity may also affect policing practices by shaping its objects of intervention, such as crime and aggression. Nikolas Rose suggests this when he argues that biocriminology has framed crime as an 'epidemic of anti-social, aggressive, and violent conduct that is thought to arise from a diminution of self-control, reasonableness, maturity, judgment, tact, and reasoning' (2000, p. 181; also see Vrecko, 2009). Standing at the intersection of science and practice, real-world experiments can thus shape objects of intervention by introducing technologies to operational sites. When this happens, ethical and practical concerns may be set aside to maintain an advantage over potential lawbreakers, as the experimental attitude implies (Koops, 2011).

In this article, I seek to learn more about the operation of real-world experiments based on a case study on an aggression detection pilot. In the pilot study, microphones recorded sounds at Bus Station S, which were analyzed for indicators of aggression. Early detection, the city's Department for Community Safety argued, could prevent violence. For the city's policymakers, the pilot study was mainly a means to discover whether this technology could help lower the number of violent incidents and improve bus drivers' perceptions of personal security.

The case opened up a set of very different issues as well. Aggression detection introduced acoustic aggression as a new object of police intervention. Yet, we should not forget that in most criminal codes, it is not forbidden to act or talk 'aggressively', although violence and insult can be offenses. At stake were the emotions one is allowed to express in public space, the authorities deciding over this, the mandate for intervention, and the method of intervention. In other words, the introduction of aggression detection includes experimenting with the legitimate use of force, a central principle of the constitutional state.

The objective of this article is to examine how an object of intervention attains its nature and status through experimentation. How is aggression constituted in the pilot study on Bus Station S?

Answering this question also serves a secondary, more general, objective: to critically engage with the format of testing at an operational site. This is relevant because the used pilot study format is increasingly credited with the production of knowledge that reflects the 'actual' performance of a technology. The core of the realworld method is to obtain knowledge that is not available in the controlled world of the laboratory (Krohn and Weyer, 1994). Yet, we may assume that some actors and events in the world outside the laboratory will be made more relevant to the outcomes than others. The question remains: which actors and events are included, and which are excluded?

I draw on ethnographic fieldwork that I conducted during the pilot. The city administration kindly allowed me to observe the pilot study for 22 months and study project documents. As part of my fieldwork, I conducted interviews with policymakers, police officers, a bus company staff member, and corporate researchers. My observations include project meetings, Community Safety meetings, a site visit to the technology supplier, observations of patrol officers, and of police control room practices. 
To understand the kind of object and how it was introduced in the pilot study, I draw on insights from material semiotics and science and technology studies (STS). This is a relational approach to the activity of experimenting. Experiments do not simply test new technologies, it argues, but require the realignment of relations between actors of various kinds, such as humans, tools, and bodies of knowledge (Latour, 1996). It is through this trial-and-error process that new objects of intervention take shape. I introduce the notion of tinkering (Knorr, 1979; KnorrCetina 1989) to the literature on real-world experiments to provide an insight into these ad hoc rearrangements.

The article shows how the technology, crime control, and acoustic spaces were tinkered with at three pilot sites: the engineer's room at Analytics Inc., the developer and supplier of aggression detection technologies; the city's Department of Community Safety; and the police control room that observed Bus Station S. A new signal of aggression was introduced to policing practices: shouts produced by the aroused and possibly violent human body. This signal did not replace the existing signals of aggression at the sites of practice. Instead, aggression as a bodily phenomenon was established as a fact to be detected in less noisy environments. The pilot study therefore introduced a norm for behavior not prescribed by criminal law, but by a set of acoustic-physical variables taken to represent a bodily state.

Through a relational approach, it thus becomes possible to study the nature of the new objects that real-world experiments introduce; who determines what they are, by which method, and what or who is excluded. We learn that experimenters make the test location selectively relevant. In this case, the pilot study location was made to represent a 'real world' that was disturbing and uncontrollable, invalidating it as a proper site for a test of technology. Thus the overarching contribution of a relational approach to real-world experimentation is that what counts as the real world should not be taken for granted, but is an outcome of the experiment.

\section{Experimenting at the Intersections of Practice, Policy, and Research}

\section{The Real-World Experiment}

The literature on real-world experiments has increasingly recognized that experiments performed in operational settings rearrange existing practices. Yet this body of work does not fully conceptualize how these rearrangements come about and to what effect. After discussing the current main insights into real-world experiments, I introduce two concepts that guide my empirical analysis: objects of intervention and tinkering.

Various authors in the social studies of science recognize that experimentation has become an institutionalized form of decision making, where policymakers and practitioners are confronted with the possibilities and uncertainties of new technologies (Gross and Krohn, 2005). This is different than attempting to learn from events that happen anyway, as is the case with the use of high-risk technologies such as nuclear power plants (cf. Krohn and Weingart, 1987); different than referring to policy as experimental because it allows for incremental innovation (cf. Dorf and Sabel, 1998); and different than stating that society-at-large is a laboratory to allude to the ways in which actors innovate, take risks, and cross pre-determined boundaries (Guggenheim, 2012). Such practices have been noted as examples of experimentation with good reason. Yet, my concern is with the practice of experimenting technology in policy and operational settings according to such formats as pilot studies and 
technology trials (Gross and Krohn, 2005). I am especially interested in such formats because they are increasingly accepted as knowledge production methods for policy.

I follow Matthias Gross and Wolfgang Krohn in identifying these deliberate, institutionalized forms as 'real-world experiments' (Gross and Krohn, 2005), previously referred to as 'real-life experiments' (Krohn and Weyer, 1994). These notions were developed in the context of knowledge society theory. In Krohn's work, the idea of the knowledge society expresses the increasing level of uncertainty about risk as a consequence of today's fast pace of innovation. Taking waste as an example of the unpredictability of the risks of new knowledge and technology, he shows how real-world experiments in waste management meet policy's need to reduce these uncertainties (Krohn, 2007). Gross argues that contemporary modes of knowledge production - problem-oriented and interdisciplinary - lead to more uncertainty. As researchers engage with the world outside the laboratory, they confront not always welcome surprises. Drawing lessons from ecological design, Gross argues that experimentation should be developed as a method 'not to overcome or control unknowns, but to live and blossom with them' (Gross, 2010, p. 45).

Although their setups differ wildly, many experimental forms have in common that they at least presuppose an organized research process that includes monitoring and evaluation (Krohn and Van den Daele, 1998). They cross institutional boundaries, involving policymakers and regulators as well as scientists, corporate researchers, and operational employees. Importantly, testing is based on the idea that the particularities of the site of implementation will reveal information about the employment of a technology that otherwise would not be available (Krohn, 2007). This is because the interactions between a technology, nature, and society are unstable, site-specific, complex, and unpredictable (Gross, 2010). The insights that pilot studies and similar formats are to deliver concern such topics as public perception, interoperability, relevant tacit knowledges, and health risks.

This body of literature also observes that real-world experiments cause controversy and friction, as participants learn by trial-and-error. Risks that would normally be avoided become part of a method with scientific authority (Krohn and Weyer, 1994). A prominent example is the experimental use of genetically-modified crops (GMOs) without conclusive evidence for health and environmental risks. In this case 'the implementation of new technology may become a means to generate exactly the knowledge that is supposed to be the basis of such implementation' (Krohn and Van den Daele, 1998, p. 4).

Taking a discursive approach, other work in STS emphasizes how real-world experiments legitimize technology implementations. With regard to EU agricultural policy, Levidow and Carr demonstrate how experimental introductions of GMOs allow risks to be scientized and shift to a language of control and measurability (Levidow and Carr, 2007). Bonneuil et al. argue that, in GMO controversies, the boundaries between laboratory and field are constantly redrawn by participants in the debate. Experiments have no intrinsic features; their locations, authorities, methods, and topics have always been subject to negotiation (2007).

The literature on real-world experiments thus informs us about the ways in which events take shape around the tension between a desire for control and a willingness to take risks. With regard to aggression detection, this can be understood as a willingness to risk false detection, illegitimate arrests, or disturbing police routines. Yet, we learn little about how objects of intervention are constituted. Experimental objects are forgotten in the literature on real-world experiments. 
Although one should be careful not to attribute intrinsic qualities to experiments and their objects, as Bonneuil et al. suggest, it may be worthwhile to question the objects of intervention that this format introduces. Once 'released', so argue Yuval Millo and Javier Lezaun, objects such as financial options contracts and GMOs cannot be withdrawn (2006). They move and mutate in their new environments, making their governability a challenge (cf. Callon, 2009). Inquiring after the kinds of objects introduced in an aggression detection pilot study therefore is relevant, especially since being aggressive is not an offense in itself. In the following, I suggest a relational approach to address the operation of real-world experiments.

\section{Experiments and Their Objects of Intervention: A Relational Approach}

I turn to insights from actor-network theory, material semiotics, and feminist STS to understand how aggression was constituted as an object of intervention. Research in these fields holds a particular sensitivity to the objects of intervention that are constituted in technological practices and the actors that are marginalized in the process ( $\mathrm{Gad}$ and Jensen, 2010). With 'object of intervention' these fields refer to the entities that come into being through practice. They are 'done', and as such, are 'simultaneously real, discursive, and social' (Latour, 1993, p. 64). Such objects range from atherosclerosis (Mol, 2002) to forensic race (M'charek, 2013) and illegal fisheries (Gad and Lauritsen, 2009).

Authors stress the relationality of these objects of intervention. It is argued that objects are always being constituted in specific arrangements of humans and things (Haraway, 1991; M'charek, 2008; Gad and Lauritsen, 2009). Aggression, for instance, is constituted by pulling together collectives of humans and nonhumans at a certain place and time, including tools, bodies of knowledge, and regulatory frameworks. Therefore, depending on the practice, aggression can be established by psychological reports, loud shouting, or the possession of firearms.

In a pilot study, the technology needs to be made to work in relation to the particularities of the site of practice to produce an object such as aggression. For the practice of policy experiments and technology trials, this means that, when a technology is introduced in an operational setting, various relations need to be reconfigured and aligned (Latour, 1988, 1996; Marres, 2012). This relational approach is different than earlier work on real-world experiments. So far, the object of intervention has at most been attributed with epistemic variability. In a study about a real-world experiment with a waste incinerator, for instance, Krohn argues that in this project "one of the concerns was to properly define the epistemic object "waste"" (2007, p. 141). This leaves unanswered the question of what comes to qualify as waste through the practice of experimentation and who decides about this.

\section{Tinkering}

A focus on how the actors tinker helps to appreciate how objects are constituted in trial-and-error situations such as real-world experiments. Karin Knorr-Cetina introduced tinkering as a concept to engage with experimenting as a creative, material, and ad hoc exercise (1979). Experimenters are tinkerers. They achieve their results given the cooperation and contestation of colleagues, opportunities in the field, and the materials at hand: 
They are aware of the material opportunities they encounter at a given place, and they exploit them to achieve their projects. At the same time, they recognize what is feasible, and adjust or develop their projects accordingly. While doing this, they are constantly engaged in producing and reproducing some kind of workable object that successfully meets the purpose they have temporarily settled on (Knorr-Cetina, 1981, p. 34).

Examples of tinkering in Knorr-Cetina's work are adjustments of research goals in the light of material opportunities and the misuse of laboratory equipment. Consequently, research products are 'multifaceted hybrids' derived from what was locally available and assumed to be significant (1979, pp. 258-259).

In material semiotic approaches tinkering is discussed in terms of the necessity to adjust relations when a technology is applied in a new setting, and frictions and disturbances occur (Mol et al., 2010). Changing a wheelchair, for instance, 'involves modulating the relationships and balancing the positions of each member of the collective' (Winance, 2010, p. 112). Following this approach, I refer to tinkering as the hands-on work of adjustment in the face of surprises, disturbances, and frictions. I diverge from previous work in material semiotics on tinkering by leaving its outcome open. In line with Knorr-Cetina's observations, tinkerers do not always aim for a balanced outcome. They may tinker according to their emerging priorities.

In my analysis of tinkering during the Bus Station S pilot study, I focus on the participants' efforts to tinker with aggression as an acoustic object (for instance, shouts or gunshots). ${ }^{3}$ I identify tinkering in the data as the changes that actors make to a technology's settings, as well as to policies, budgets, and work spaces. In particular, I examine the moments when actors tinkered to distinguish foreground and background sounds. Foregrounding a sound is to turn it into a signal for action, and therefore an object of intervention, in contrast to background noise (Latour and Woolgar, 1986). This analysis uses my informants' understanding of aggression, signals, and noises, as I am interested in aggression as an object constituted by their activities.

To sum up, I suggest a relational approach to understand how a new object of intervention is introduced in a real-world experiment. Objects of intervention, such as aggression, do not exist on their own; they are made relevant through experimentation in terms of their relations in human and non-human collectives. I introduce tinkering as a notion through which we can understand how objects are constituted in trial-anderror processes characteristic of real-world experiments. Studying tinkering, we learn about the realignments that constitute aggression at the test site and therefore about the nature of this new object. In the case of aggression detection, this refers to whom or what identifies aggression, on what basis, and how it can be intervened with. For instance, aggression as a physical state is intervened with differently than aggression established by a criminal track record.

This analytical framework enables me to examine how aggression is brought into being in a process that includes surprises, friction, and disturbances. At the same time, this article shows that not all actors causing surprises and disturbances are equally relevant to the detection of aggression. So even though the format of realworld experimentation is given the capacity to generate more genuine insights into local realities, 'the real world of practice' (including its disturbances) should not be taken for granted (cf. Ingram, 2012). It is both an outcome and an instrumental part of 
experimenting. To make this argument, I first turn to the case study: the pilot study at Bus Station S.

\section{Detecting Aggression in the Absence of a 'Ground Truth'}

\section{Bus Station $S$}

Bus Station $\mathrm{S}$ was one of the first pilot studies in the Netherlands to test the acoustic detection of aggression in an operational setting. Installed for one year, seven microphones detected the sound of vocal aggression. When it was detected, the police officers in the nearby control room would first be alerted by the sound of an artificial trumpet. Next, the surveillance cameras at Bus Station $\mathrm{S}$ would turn to the originating location of the sound. Bus Station S would subsequently feed live audio and video directly from to the control room for 60 seconds. ${ }^{4}$

The project was initiated and funded by a regional government agency for the organization of public transport. This agency asked a local city administration to host and manage the pilot study. The host city's project manager cooperated closely with the police station that had installed the technology in its control room. A company that I call 'Analytics Inc.' provided, installed, and fine-tuned the technology. Bus passengers were not told about the use of aggression detection and were therefore not actively involved in this pilot study.

The regional government's primary aim was to learn whether sound detection could reduce the number of violent incidents and improve the bus drivers' perception of personal security. The host city suggested Bus Station $\mathrm{S}$ as the test location because it was known as a site where bus drivers and passengers were frequently harassed. In my conversations with local police officers, they described the station as 'old, dark, and grimy', and therefore vulnerable to criminal activity. ${ }^{5}$ Most of its reputation, however, had built up in previous years. Local newspapers, police officers, and policymakers suggested that the source of the problem was a nearby nightclub. This club, it was assumed, attracted a crowd that often acted under the influence of alcohol and drugs. But since the nightclub had closed down, the area had calmed down in the eyes of the police. For the city's project manager, however, Bus Station S was still an area of concern. She believed that passengers on their way to other nightlife districts could be a source of nuisance.

\section{Modeling Aggression}

Analytics Inc. operates in the field of artificial intelligence. To analyze sounds, it applies insights from biology and cognitive science. The company decided to focus on the security market when aggression became a central topic of Dutch public debate and an issue of increasing importance on corporate and government agendas. Measuring aggression, however, is not straightforward. In fact, Analytics Inc.'s experts argue in a conference paper ${ }^{6}$ that there is no absolute scientific measure for how much aggression is reflected in a voice. In the following, I first take a closer look at the research behind this system. This background will be helpful in understanding how the participants in the pilot study tinkered, and what they produced.

'Ninety percent of all physical violence is preceded by aggressive speech', so Analytics Inc. argued in its sales presentations. In the absence of an absolute 
definition of aggression, this company claims to have modeled what humans perceive as aggressive. From a master's thesis that did much of the groundwork for this technology, we learn that this system is based on a three-step model of human perception of emotions through hearing. First, foreground and background sounds are separated. Next, the human brain analyzes vocal cues from other persons that 'betray' aggression. These vocal characteristics 'attract human attention'. Finally, values are assigned to the cues to express the level of aggression: irritation, anger, and rage.

The cues for the system were determined by applying research that describes the production of sounds by humans that attract the human attention. In a state of anger or panic, Analytics Inc. argues, high blood pressure and muscular tension affect the operation of the vocal chords. Consequently, the voice is marked by aggression. To approach these cues, however, the company resorted to studies of the behavior of vocal chords when humans raise their voice to attract the attention of others, irrespective of their emotional state. These are quantified values of volume, pitch, and quality (Analytics Inc. mentions harshness as an example of quality). The machine measures these characteristics; it does not analyze the content of the words and sentences that it records.

Analytics Inc.'s researchers did not consider this model of aggression to set an absolute measure. Sounds have a different character in every new environment, they argue, and a machine cannot distinguish between a human voice and other sounds on its own. This is the 'signal-to-noise paradox' (doctoral dissertation; master's thesis). The paradox is that the system needs to correctly analyze a signal, but it can only find the signal on the basis of information about what a correct analysis is. Thus, Analytics Inc. needs pilot studies to teach the system what constitutes a correct signal, and what constitutes background noise for every location. Consequently, as the next section demonstrates, aggression as an acoustic object is different for every new location.

\section{The Signal of Aggression: Three Sounds}

In my discussion of the Bus Station S pilot study, I examine aggression as three different acoustic objects at locations belonging to the experimental setting: Analytics Inc.'s headquarters, the Department of Community Safety at city hall, and the police control room near Bus Station S. For each location, I describe how the actors tinkered to constitute aggression as a foreground signal, and how this involved realignments between various actors (Winance, 2010).

\section{Aggression as Shouts}

The first location we visit is Analytics Inc.'s headquarters. Shortly after its installation at Bus Station S, the system settings required adjustment because seagulls, ambulance sirens, the wind, and heavy engines caused false alarms. I used a fieldwork visit to Analytics Inc.'s headquarters and interviews with a researcher and one of the system's developers to learn what tinkering can mean to aggression as an intervention object.

Analytics Inc. referred to tinkering as 'fine-tuning'. Fine-tuning the equipment 'is not what I maybe think it is', one of Analytics Inc.'s researchers told me when I visited the company. 'We don't go to the location, turn on a switch and immediately see the effect.' Analytics Inc. fine-tunes the technology from its headquarters, far removed from Bus Station S. Here, the company receives an audio stream from the 
on-site installation. This allows it to check the equipment in real-time for false alarms. To explain, the researcher pointed to a long list of acoustic-physical variables on a computer screen, such as pitch, duration, and energy. This is Analytics Inc.'s coding system for aggression. The system operates as a filter that separates what the company considers foreground signals from background noises. ${ }^{7}$ Fine-tuning the system entails changing the thresholds of these filters, the values of the variables that trigger an alarm.

The filters' settings are thus Analytics Inc.'s tools for tinkering with the system. Let us first take a closer look at the development of these filters to understand what types of foregrounds and backgrounds they distinguish. One of the developers of the system started out describing his research by stating that sound is simultaneously fascinating and complex, and this is what made his work both trying and intriguing. Filters cannot be standardized because the acoustic-physical traits of sounds and the meanings we give them vary endlessly, he explains. For example, in terms of pitch and energy the sound of an ambulance siren at one hundred meters distance is very different than the same sound from ten meters away. Humans know the same object produces this sound, but a machine does not.

A system must thus be taught the precise distinctions between foreground and background sounds. The Analytics Inc. research team developed a filter to detect the human voice as a foreground signal; the aggression filter that should trigger the alarm. A second filter for monotonous noise was added to mark background sounds that should not be analyzed as aggression. Despite this filter, the number of false alarms remained high. Therefore, the researchers decided to add a third set of filters; what I term noise filters. They detect specific common urban background noises, such as scooters. Once the noise filters detected a specific sound, it could no longer be marked as aggression.

In my conversations with Analytics Inc.'s experts, they found it difficult to describe how exactly the filters are fine-tuned. They said fine-tuning is a 'special skill' or 'trick of the trade' that is particularly hard to explain to outsiders. An expert 'sits down with headphones on, changes one of the settings, sees what happens, and tries it again'. The researcher continues:

This is our expertise, to learn from, well, if I change these parameters, then there'll be too many false alarms and if I change that one, shut it down, so to speak, I'll get too few alarms. So, you need to find the balance. This is where your skill comes in ... So, for frequencies, above what level is it interesting? Below what does it stop being interesting? And how often should a sound happen, before you mark it as aggressive?

Site recordings are played (with) repeatedly to find a balance between false positive alarms and false negative alarms (incidents that the system misses).

What exactly happened to the settings at Bus Station S remains confidential. We learn, however, from what did not happen as part of this effort to fine-tune. This tells us that Analytics Inc. tinkered with the aggression filter to exclude non-human sounds; the engineers focused on the human voice. The point is relevant as Analytics Inc. also developed systems to recognize the breaking of glass or gunshots. Moreover, as I learned by joining patrolmen on their beat in the area surrounding Bus Station $\mathrm{S}$, sounds other than shouts can be a signal of aggression. Some officers, for instance, are especially attentive to barking dogs, which were excluded from the system. This made the acoustic signal of aggression at Bus Station $\mathrm{S}$ exclusively human. 
Not only did Analytics Inc. develop a filter that only included the human voice, but it also needed to be a loud voice: shouting. Low-voiced threat, for instance, was excluded. This can be related back to the technology's purpose: the detection of verbal arguments in nightlife situations. The previous section showed Analytics Inc. defined the markers on the basis of research that describes the sounds that people make when they aim to attract attention, irrespective of emotional state. The markers were thought to function well precisely because the system was not intended to detect soft-spoken aggression as this was not considered an indicator of nightlife violence.

It should also be noted that a procedure that was part of earlier studies by Analytics Inc. was skipped at Bus Station S. Analytics Inc. had previously invited their clients, the police for example, to listen to recordings of aggression, and asked them to mark the level of aggression on a scale from 1 (requiring immediate action) to 3 (a false alarm) (conference paper, Analytics Inc.). This means that at Bus Station S, Analytics Inc.'s experts had an important role in determining what counted as human aggression.

Foreground signals and background noises were the outcomes of tinkering rather than pre-givens (Latour and Woolgar, 1986). Tinkering involved the inclusion of some sounds, at the expense of others. This part of the pilot study was mainly an effort to exclude non-human producers of sound, such as dogs, from what was to become a signal for aggression. Accordingly, a distinctively human aggression became a foreground signal. This was not any kind of human aggression, such as an interaction between two persons or a soft-spoken threat. The signal foregrounded by Analytics Inc. was a loud fight. As we learned in the previous section, aggression was initially conceptualized as the outcome of the physical processes in an aroused body. Analytics Inc. translated this knowledge from the field of auditive cognition to a notion of aggressors as loud nightlife visitors.

\section{Aggression as Bus Horns}

A second signal for aggression was suggested in an early phase of the pilot study, as I learned from retrospective interviews conducted a few months into the pilot. Here I examine the foregrounding and backgrounding of this second signal to understand the realignments the city's Department of Community Safety made through tinkering.

To mark the formal start of the pilot study, Analytics Inc. demonstrated the system at Bus Station S and in the police control room. Normally, the bus companies were not consulted, but, for this occasion, a few staff members were invited to witness the operation of the system. During the demonstration, they sat in the police control room where they saw and heard how recordings of aggressive shouting that Analytics Inc.'s employees played on the bus platform triggered the system. One of the bus company's staff pointed out a problem after the demonstration. As he put it:

The system only works when people argue with shouting or fighting on the platform. But what if something happens on the bus? The system does not notice that. Then how can the driver attract attention?

He then asked the city's project manager: would it be possible for the alarm to be triggered by the bus horn? Among the bus drivers in this region, the horn is a wellknown signal of distress, he told her. To blow the horn three times means you are in 
trouble and need the assistance of other bus drivers. He suggested that this could be a signal for acoustic detection.

The city's project manager thought the suggestion was interesting, so she asked Analytics Inc. for a quote. Modeling the bus horn did indeed seem possible to Analytics Inc. It had developed filters to detect mechanical sounds before, such as gunshots. Horns were already part of the noise filter, but the filter could be adjusted to mark three consecutive blows as a signal of aggression. Thus, instead of background noise, the bus horn would be a foreground signal.

However, when Analytics Inc. returned with a price estimate, the city's project manager felt that the cost was too high. There was a limit to the amount of money she could spend on innovations to benefit the private sector (Dutch regional bus services were privatized in 2000). This was not just a decision about financial responsibilities in crime control but also about who would intervene with aggression. If a bus driver uses the horn, it means there would be other drivers in the vicinity, the project manager argued. In such cases, drivers should first attempt to solve the problem among themselves. 'We [project manager and bus company's staff member] talked about it, and if you use the horn, there are buses nearby. So maybe your colleagues can help you.'

The project manager's actions can be understood as tinkering a signal to the background. Were the sound detection system to detect a bus horn as aggression, this would mean that misbehavior would not be constituted just by knowledge of cognitive-physical processes in the human body. Instead, what the bus drivers experience as aggression would lead to an alarm. However, the bus horn was excluded from the order of intervention at Bus Station S. This positioned aggression as a set of acoustic-physical variables produced by an aggressor at the core of the order of intervention enacted by the city at that moment.

Accordingly, tinkering not only involved Analytics Inc.'s employees fiddling with the settings of the sound filters. It also included 'modulating the relationships and balancing the positions of each member of the collective' (Winance, 2010, p. 112). If the bus horn were deemed a signal of intervention, it would establish a direct relation between the driver inside the bus and the local police control room. This relation did not exist as yet, as bus companies were held responsible for maintaining order on their own terrain. Buses were equipped with a distress button that connects them to the bus companies' control room, from where the police can be contacted. The buses and bus platforms, however, were the property and responsibility of the bus companies. Excluding the bus horn as a signal of intervention therefore re-established intervention inside the bus as the drivers' responsibility.

\section{Aggression as Sirens/Silence}

The last acoustic object I discuss is the siren. It caused numerous false alarms in the police control room. In this section, I use observations of everyday practices in the control room and interviews with police officers to show how the officers tinkered with acoustic space, including the sound detection system, to manage these false alarms.

The control room is the operational center for the orchestration of police action in the district. It is where police reports about incidents reach the station by phone, portophone (walkie-talkie), and digital messaging. The control room operators emphasized that their main responsibility is to care for their colleagues on patrol: 'We 
are our colleagues' security,' said one operator. 'You always need to be alert to help them.'

This became evident in their daily activities. From the control room, the officers arranged for backup if necessary. They also provided patrolmen with information about individuals living at an address and about the histories of vehicles and their owners. Before a patrolman made a house call, his or her control room colleagues checked for previous offenses, possession of firearms, and previous confrontations with the police. 'If we know an aggressive person lives at that address we can tell them [patrolmen] to take a car with them,' an operator explained.

During the pilot study, the control room operators tinkered to manage a sound that was unintentionally marked as aggression: the sirens of emergency vehicles. Bus Station $\mathrm{S}$ is located on a road to a hospital and a fire station, so sirens triggered at least one false alarm each day. This problem could not be solved by Analytics Inc. The company argues in a conference paper that the acoustic-physical characteristics of a siren are similar to those of an aggressive voice; ironically, both attract human attention. A siren of a passing ambulance will therefore generate an alarm. The sirens could not be sifted out with a separate noise filter, one of the system's developers explained in an interview, because by sifting out sirens, vocal aggression would also be sifted out. The latter could thus be recorded without triggering an alarm, and this was a risk that Analytics Inc. was not willing to take.

To cope with the false alarms, the police officers tinkered with the sound detection system. This was done in the context of the everyday operations of adjusting the acoustic space. To make clear how this is relevant, I first describe the role of sound in the daily operations of this police control room. In this environment, policemen use sound to distinguish relevant signals. They can do so because they are trained listeners. A regular officer can do an interview, listen to his or her colleagues, and tune into a portophone call all at the same time. In the control room, operators listen for computer bleeps that report incidents. Also, they have learned to extricate relevant messages from an uninterrupted flow of spoken portophone calls by listening for regional codes. Another way of assigning relevance is to judge the level of panic or distress in the voices of their colleagues on the phone. Sometimes it is very clear that a patrolman is in trouble because he or she will use the phrase for emergencies, 'assistentie, collega' ('help colleague'). However, a policeman who has just joined the force, an operator claimed, might sound panicky for no reason, so 'you have to know who you are talking to.'

Whenever I visited the control room, the officers incessantly adjusted the various technologies that transmit audio. The operators did this to manage their individual attention to calls. For instance, some officers turned the volume of incoming portophone messages up and down several times during a shift. If a phone call or portophone message was inaudible, the patrolman on the other end of the line was asked to move position or to clear the line. Also, the television and radio were often on, but at times an operator would decide to lower the volume. An officer explained, 'Some colleagues leave the TV on all day, but I can't do that anymore.' She meant that her hearing had become less sharp over the years. In addition, the operators managed the physical connection of the control room to other parts of the police station. They closed and opened the doors and windows according to the level of disturbance from the outside world.

Coming back to aggression detection, in this environment, at first the loudspeakers that transmitted the aggression alarm were kept in the basement of the police station. The control room manager was worried by the disturbance false alarms 
could cause. After six months, they decided to place the speakers in the control room. 'Here's hoping for the best,' the control room manager said shortly after the speakers were installed, 'but it's a pilot study, and we need to try.'

Once installed in the control room, false aggression alarms transmitted the sound of sirens that triggered the alarm. This was especially disturbing if an emergency vehicle was heading to an accident close by because it required the operators' full attention. The police reacted to false alarms by lowering the volume or disconnecting the speaker wires. At the start of a new working day, the volume was turned up again or the wires were reconnected. After a couple of months, however, the effort to reconnect the technology stopped. Starting out as an unintentional foreground signal in the control room, ultimately the sound detection system was silenced.

The operational domain of the pilot study thus demonstrates another way in which people tinker: by regulating acoustic space. The police officers tinkered with the system to make sure that operations in the control room ran smoothly. Thereby they re-established the existing order of intervention, accompanied by acoustic signals such as computer bleeps in the control room.

By tinkering with aggression detection the officers foregrounded aggression as a different sound object than Analytics Inc. had introduced. They established aggression in the control room on the basis of the experience of officers on patrol, identifiable by their voice or by the computer bleeps of the digital messaging system. In addition, records of previous offenses were drawn together to establish a risk of aggression. Whereas Analytics Inc. located aggression in the body of the supposed aggressor, the control room operators located aggression in records of previous events and the experience of their colleagues.

Tinkering thus involved establishing different relations. In line with KnorrCetina's findings, tinkering was done in line with the actors' (emerging) priorities $(1979,1981)$. In this case, the officers in the control room reset their priority to care for their colleagues on patrol, thereby negating the direct link between the microphones at Bus Station S and the police control room. Furthermore, the officers on the street and in the control room were reconstituted as the authorities for establishing aggression. Silencing the detection system also reinstated the temporality of control room practices. If possible, the operators informed patrolmen about individuals, households, and vehicles at least five minutes before they approached them to make sure they were prepared. Sound detection, on the other hand, was communicated by live recordings on-site. Accordingly, police officers were expected to respond to an alarm when aggression was supposedly already happening.

The enactment of a local work order based on other acoustic signals eventually silenced the detection system. Nevertheless, Acoustic Inc.'s aggression was not subdued entirely, as I suggest in the next section.

\section{The Aggression Moment}

Something else is worth noting about the police's tinkering in the control room. Three months after the formal start of the pilot study, the control room manager began expressing his disappointment in the performance of the technology, and he did so repeatedly on my following visits. Surprisingly, the police carried on tinkering with the acoustic space. They decided to move the speakers from the basement to the control room, and for several months after repeatedly reconnected the wires after disconnecting them. Why reconnect speakers when the sound of aggression detection 
disturbed the control room operators? As argued above, the police tinkered to retain their work practices. Yet, it seems like tinkering served another purpose as well.

Connecting and reconnecting the system seemed to be aimed at generating false alarms that demonstrated failure. ${ }^{8}$ Yet, this was not taken to be a failure of the technology. Instead, the location was performed as an erratic and uncontrollable reality. We read this in the evaluation report based on the police's experience with aggression detection. After one year of testing, the evaluation committee stated that the device had not detected aggressive behavior in the trial period. ${ }^{9}$ The ensuing evaluation report was brief on the outcome: 'aggression detection does not work on this busy road with buses, emergency services, and other sources of sound. The evaluation report attributed the problem of false alarms not to the technology, but to the location. In particular, it was attributed to local actors that produce noise.

Thus, tinkering characterized the location as noisy, unpredictable, and uncontrollable. This is in contrast to the potential performance of the location of practice as predictable, routine, and controllable. ${ }^{10}$ It had two advantages for the police. First, they could argue that the technology needed to be removed from this site. Second, they did not need to discuss the technology itself in detail to make this argument.

By suggesting that the test site disturbed the detection of aggression, the actors separated the object of aggression from the site. In other words, aggression was constituted as a detectable object (just not at Bus Station $\mathrm{S}$ ). This allowed the city to consider testing this technology at another location. Furthermore, it allowed Analytics Inc. to specialize in indoor locations, making outdoor sounds a disturbance to detecting the fact of aggression.

Therefore, aggression as a trait of an aroused body translated into a set of acoustic-physical variables was left uncontested, even if the technology produced false alarms. In fact, Analytics Inc.'s aggression became part of the parlance of the policy makers and the police. In the evaluation meetings and in interviews after the pilot study, policy makers and police spoke of 'an aggression' as a discrete, measurable moment. One of the policy officers of the city described the technology's future operation as 'when the camera turns to an aggression moment, a police officer will notice this, and he will be able to instruct his colleagues right away.'

\section{Conclusions}

I began this article with the following problem: real-world experiments that test new technologies can affect policy and practice by introducing new objects of intervention. This raises practical and ethical concerns, as a focus on potential technological advantages can overshadow careful scrutiny of the objects, policies and practices introduced through experimenting. We should therefore ask how objects of intervention attain their nature in real-world experiments. The aggression detection pilot study helps answer this question as it introduced acoustic aggression as an object of intervention. The Dutch criminal code does not forbid 'aggressive' speech and behavior, yet in this case a technology was introduced that reported aggression to the police. At stake, therefore, were the emotions one is allowed to express in public space, the authorities deciding over this and the method of intervention.

In real-world experiments, objects of intervention attain their nature through tinkering. This article discussed three acoustic signals of aggression established by the tinkering activities of engineers, policymakers, and police officers. The object of 
aggression introduced by Analytics Inc. was a shout produced by the aroused human body, in particular that of the nightlife visitor. But in city hall, aggression also took on the form of bus horns activated by threatened bus drivers. In the police control room, phone conversations and computer bleeps notified police officers of risky persons and situations.

The participants tinkered by adjusting the aggression detection device, roles in crime control, and the acoustic space of the control room. Tinkering, this case study shows, is a relational effort with the effect of including some actors, while marginalizing others. At the offices of Analytics Inc., foregrounding aggression as shouts required excluding mechanical and animal sounds as indicators of aggression. At the city's Department of Community Safety, the police's involvement with the bus drivers' experience of aggression was cut short by discarding the bus horn as a signal of aggression. In the control room, police officers tinkered to silence the aggression detection alarm. Thus, they foregrounded the messages of fellow patrolmen, and backgrounded the events at Bus Station S.

Tinkering at the police control room was not only a matter of realigning work practices. The aggression detection system in the control room was repeatedly connected and disconnected to demonstrate the number of false alarms generated. These were not attributed to the technology or the signals of aggression that it foregrounded, but to the noisiness of the test site. Thereby, the test site was disproved, not Analytics Inc.'s aggression (aggression as shouts produced by an aroused body) as an object of police intervention.

To conclude, a relational approach to real-world experiments firstly shows that a technology experiment can introduce objects for intervention. Focusing on the relational aspects of tinkering allowed me to study the nature of these new objects; who determines what they are, by which method, and what or who is excluded. Aggression was shifted in and out of the human body, depending on the technology and knowledge applied. Even though the technology's developers started out with a notion of aggression as a receiver's bodily experience, Analytics Inc. put in practice aggression as shouts produced by the bodies of nightlife visitors. Despite the fact that this object was tinkered away in the control room, it continued to exist as an 'aggression moment' in the policy realm. The pilot study therefore introduced a norm for behavior not prescribed by criminal law, but by the acoustic-physical variables understood to represent bodily aggression.

Secondly, this approach made it possible to critically engage with the format of experimenting at a location of practice. The pilot study format is credited with the production of knowledge that reflects the 'actual' performance of a technology. Yet, in tinkering processes, actors and events at the pilot study location are only selectively made relevant. Dogs and bus drivers were disturbing uninvited guests, instead of actors to be included in an aggression alarm.

The overarching contribution of this article to studies of real-world experiments is that what counts as the real world cannot be taken for granted. Matthias Gross heads in this direction when he argues that in experimental projects outside the laboratory events are only surprising in the light of (reconstructed) expectations (2010). The argument made here is slightly different. Pilot studies, trials, and tests are formats which produce particular accounts of reality together with accounts of technology. A relational focus on tinkering shows that, in each real-world experiment, the site of implementation would be made relevant differently. The case of aggression detection demonstrates that how exactly the 'real world' is made 
relevant needs to be considered as it has an effect on the truth status of the introduced objects of intervention.

\section{Notes}

${ }^{1}$ Fictitious names guarantee the anonymity of my informants, with the exception of the Dutch Data Protection Authority (Dutch DPA).

${ }^{2}$ See, for instance, Gates (2010); Boyle and Haggerty (2009); and Helten and Fischer (2004).

${ }^{3}$ The material production and consumption of sound is the focus of the social studies of sound (Pinch and Bijsterveld, 2012). This field has a particular interest in the materiality of sound, and its embeddedness in society, science, and technology (Pinch and Bijsterveld, 2004). Authors in this field have shown the role of sound practices in the shaping of subcultures and identities (Waksman, 2004), and demonstrated that 'sounds are places where power relations are managed, elaborated and acted out' (Sterne, 2003, p. 59). This body of work has not, however, explicitly addressed the bringing into being of acoustic objects in material semiotic terms. I turn to other STS literatures to describe how aggression is established as an acoustic object. I should mention here that some authors' work does point in this direction. For instance, Mody (2005) shows how sounds are part of the organization of laboratory work. Lachmund (1999) examines how lung sound codification systems are variably constituted in different countries. ${ }^{4}$ The Dutch DPA contended that no privacy regulations were violated. It allowed these practices because Analytics Inc. claimed that it did not save recordings beyond the 'purposes of the experiment', and because the content of words and sentences was not analyzed (letter, Dutch DPA 2005).

${ }^{5}$ All quotes are translated from Dutch by the author.

${ }^{6}$ Citing the full titles of the research literature and policy documents that I quote or reference in this article could reveal the names of individuals, locations, or organizations. I have therefore chosen to mention only the document types in the text, and exclude those references from the bibliography.

${ }^{7}$ I follow my informants' terminology in referring to codification systems as filters.

${ }^{8}$ The demonstration function of experiments is well described in STS. See, for instance, Shapin and Schaffer (1985), Latour (1988), Barry (2001), Simakova (2010). Whereas most of these accounts demonstrate success, in this case the actors seem to demonstrate failure.

${ }^{9}$ Here I do not discuss the technology's performance in terms of missed and detected cases of aggression because the police do not register all cases of verbal and physical violence.

${ }^{10}$ For an overview of tropes on everyday practice, see Elias 1998 [1978].

\section{References}

Barry, A. (2001) Political machines: Governing a technological society (London and New York: Athlone Press). 
Bonneuil, C., Joly, P. and Marris, C. (2007) Disentrenching experiment: The construction of GM-crop field trials as a social problem Science, Technology \& Human Values, 33(2), pp. 201 - 229.

Boyle, P. and Haggerty, K.D. (2009) Spectacular security: Mega-events and the security complex, International Political Philosophy, (3), pp. 257-274.

Callon, M. (2009) Civilizing Markets: Carbon trading between in vitro and in vivo experiments, Accounting, Organizations and Society 34, pp. 535-548.

Dorf, M.C. and Sabel, C.F. (1998) A constitution of democratic experimentalism, Columbia Law Review 98(2), pp. 267-473.

Elias, N. (1998) On the concept of everyday life, in: J. Goudsblom and S. Menell (Eds) The Norbert Elias Reader (Oxford and Malden, Mass.: Blackwell Publishers), pp. 166-174.

Gad, C. and Jensen, C. B. (2010) On the consequences of post-ANT, Science, Technology \& Human Values, 35(1), pp. 55-80.

Gad, C. and Lauritsen, P. (2009) Situated surveillance: An ethnographic study of fisheries inspection in Denmark, Surveillance and Society, 7(1), pp. 49-57.

Gates, K. (2010) The Tampa "smart CCTV" experiment, Culture Unbound, 2, pp. 6789.

Gross, M. (2010) Ignorance and surprise: Science, society, and ecological design (Cambridge, MA: MIT Press).

Gross, M. and Krohn, W. (2005) Society as experiment: Sociological foundations for a self-experimental society, History of the Human Sciences, 18(2), pp. 63-86.

Guggenheim, M. (2012) Laboratizing and de-laboratizing the world: Changing sociological concepts for knowledge production, History of the Human Sciences 25, pp. 99-118.

Haraway, D. (1991) Situated knowledges: The science question in feminism and the privilege of partial perspective, in: Simians, cyborgs, and women. The reinvention of nature (London: Free Association Books), pp. 183-202.

Helten, F. and Fischer, B. (2004) Reactive attention: Video surveillance in Berlin shopping malls, Surveillance and Society, 2(2/3), pp. 323-345.

Ingram, M. (2012) Real-world experiments in ecological restoration, Science as Culture, 21(4), pp. 582-586.

Knorr, K. (1979) Tinkering towards success: Prelude to a theory of scientific practice Theory and Society, 8(3), pp. 347-376. 
Knorr-Cetina, K. (1981) The manufacture of knowledge: An essay on the constructivist and contextual nature of science (Oxford: Pergamon Press).

Koops, B.J. (2011) The evolution of privacy law and policy in the Netherlands, Journal of Comparative Policy Analysis: Research and Practice, 13(2), pp. 165179.

Krohn, W. (2007) Nature, technology, and the acknowledgement of waste, Nature and culture, 2(2), pp. 139-160.

Krohn, W. and Van den Daele, W. (1998) Science as an agent of change: Finalization and experimental implementation, Social Science Information Sur Les Sciences Sociales, 37(1), pp. 191-222.

Krohn, W. and Weingart, P. (1987) Nuclear-Power as a social experiment - European political fall out from the Chernobyl meltdown, Science Technology \& Human Values, 12(2), pp. 52-58.

Krohn, W. and Weyer, J. (1994) Society as a laboratory: The social risks of experimental research, Science and Public Policy, (21), pp. 173-183.

Lachmund, J. (1999) Making sense of sound: Auscultation and lung sound codification in nineteenth-century French and German medicine, Science, Technology, and Human Values 24, pp. 419-450.

Latour, B. (1988) The pasteurization of France (Cambridge, Mass. and London: Harvard University Press).

Latour, B. (1993) We have never been modern (Cambridge, Mass.: Harvard University Press).

Latour, B. (1996) Aramis, or, The love of technology (Cambridge, Mass. and London: Harvard University Press).

Latour, B. and Woolgar, S. (1986) Laboratory life. The construction of scientific facts (Princeton, New Jersey: Princeton University Press).

Levidow, L. and Carr, S. (2007) GM crops on trial: Technological development as a real-world experiment, Futures, 39(4), pp. 408-431.

Lezaun, J. and Millo, Y. (2006) Regulatory experiments: GM crops and financial derivatives on trial, Science and Public Policy, 33(3), pp. 170-190.

Marres, N. (2012) Material participation: Technology, the environment and everyday publics (Basingstoke: Palgrave Macmillan).

M'charek, A. (2008) Silent witness, articulate collective: DNA evidence and the inference of visible traits, Bioethics 22(9), pp. 519-528. 
M'charek, A. (2013) Beyond fact or fiction: On the materiality of race in practice, Cultural Anthropology, 28(3), pp. 420-442.

Mody, C.M.C. (2005) The sounds of science: Listening to laboratory practice, Science, Technology, and Human Values 30(2), pp. 175-198.

Mol, A. (2002) The body multiple: Ontology in medical practice (Durham and London: Duke University Press).

Mol, A., Moser, I. and Pols, J. (Eds) (2010) Care in practice. On tinkering in clinics, homes and farms (Bielefeld: Transcript).

Pinch, T.J. and Bijsterveld, K. (2004) Sound studies: New technologies and music, Social Studies of Science 34(5), pp. 635-648.

Pinch, T.J. and Bijsterveld, K. (Eds) (2012) The Oxford handbook of sound studies (New York and Oxford: Oxford University Press).

Rose, N. (2000) The biology of culpability: Pathological identity and crime control in a biological culture, Theoretical Criminology, 4(1), pp. 5-34.

Shapin, S. and Schaffer, S. (1985) Leviathan and the air-pump: Hobbes, Boyle, and the experimental life (Princeton, N.J.: Princeton University Press).

Simakova, E. (2010) RFID 'Theatre of the proof': Product launch and technology demonstration as corporate practices, Social Studies of Science, 40(4), pp. 549576.

Sterne, J. (2003) Headset culture, audile technique, and sound space as private space, Tijdschrift voor Mediageschiedenis, 6(2), pp. 57-82.

Vrecko, S. (2009) Therapeutic justice in drug courts: Crime, punishment and societies of control, Science as Culture, 18(2), pp. 217-232.

Waksman, S. (2004) California noise: Tinkering with hardcore and heavy metal in Southern California, Social Studies of Science, 34(5), pp. 675-702.

Winance, M. (2010) Care and disability. Practices of experimenting, tinkering with, and arranging people and technical aids, in: A. Mol, I. Moser and J. Pols (Eds) Care in practice. On tinkering in clinics, homes and farms (Bielefeld: Transcript), pp. 93-117. 
